\title{
Potentiality of curcumin on ISHAK scoring system and the expressions of BAX, IL-17A, and EGF in the treatment of Schistosoma mansoni infection using Swiss albino mice
}

\author{
El Saftawy, E.A. ${ }^{1,2}$, El-Dardiry, M.A. ${ }^{3 *}$, Abd-Elaal, A.A. ${ }^{4,5}$, Negm, M.S.I. ${ }^{6}$, Amin, N.M. ${ }^{1,7}$ \\ ${ }^{1}$ Medical Parasitology Department, Faculty of Medicine, Cairo University, Cairo, Egypt \\ ${ }^{2}$ Medical Parasitology Department, Faculty of Medicine, Armed Forces College of Medicine, Cairo, Egypt \\ ${ }^{3}$ Medical Parasitology Department, Faculty of Medicine, Fayoum University, Cairo, Egypt \\ ${ }^{4}$ Medical Parasitology Department, Faculty of Medicine Kasr Al-Ainy, Cairo University, Giza, Egypt \\ ${ }^{5}$ Postgraduate Department, Armed Forces College of Medicine (AFCM), Cairo, Egypt \\ ${ }^{6}$ Pathology Department, Kasr Al-Ainy School of Medicine, Cairo University, Egypt \\ ${ }^{7}$ Master's degree of clinical pathology, Cairo University, Cairo, Egypt \\ *Corresponding author: marwaahmed2008@outlook.com
}

\section{ARTICLE HISTORY}

Received: 24 October 2021

Revised: 14 December 2021

Accepted: 15 December 2021

Published: 20 February 2022

\begin{abstract}
The present study evaluated the antiparasitic effect of curcumin extract on Schistosoma mansoni in Swiss albino mice. The experimental design included four groups of S. mansoniinfected mice; without treatment (controls), curcumin-treated, Praziquantel (PZQ)-treated, and PZQ +curcumin treated mice. The results showed that curcumin improved ISHAK confluent necrosis score up to zero. PZQ +curcumin showed a significant reduction in portal inflammation. Both activity and fibrosis demonstrated lower scores in all treated groups, however, PZQ revealed a marked increase in confluent necrosis and interface hepatitis. Besides, the lobular inflammation revealed worsening in the overall ISHAK score in all treated groups compared with the control. Few periocular granulomas were recovered by PZQ +curcumin treatment at day 35 post-treatment $(6 \pm 1.2), P$-value $<0.05$. Curcumin revealed a mild reduction $(60 \pm 7.376)$. Curcumin-treated groups, with and without $P Z Q$, resulted in higher significant Immunoreactivity score (IRS) for Bcl-2-associated X (BAX) and lower Interleukine17A (IL-17A), and Human epidermal growth factor (EGF), compared to the control. However, PZQ revealed a lower mean IRS value in BAX, higher IL-17A and EGF in the periovulatory granuloma. It was concluded that $\mathrm{PZQ}+$ curcumin treatment had a potent synergistic outcome through lessening the number of granulomas, the inflammatory events, and the expression of EGF, and amelioration of apoptosis in the periovulatory granulomas if compared with either PZQ or curcumin alone.
\end{abstract}

Keywords: BAX; EGF; IL-17A; Curcumin; ISHAK score.

\section{INTRODUCTION}

Schistosomiasis is one of the major tropical diseases, it causes 200 million infections in sub-Saharan Africa, South America, and a wide zone of Asia with three times that many at-risk (Kabatereine et al., 2004; Alemayehu et al., 2017; Tefera et al., 2020). The three main species of Schistosoma that cause disease in man are $S$. mansoni, S. hematobium, and $S$. japonicum (Alzaylaee et al., 2020). An estimated percentage of $0.1 \%$ of those patients, i.e., nearly 200,000 , die annually due to this disease while more than $10 \%$ of infected people suffer from the chronic damaging sequels of the disease to the vital organs precisely the liver in Schistosoma mansoni and $S$. japonicum and kidney in S. haematobium. Infection with the parasite occurs mainly through the penetration of cercariae that emerged formerly from the freshwater snails (King, 2010; Zhao et al., 2019; Mawa et al., 2021). The schistosomulum reaches the portal vein via the blood circulation to become adult worms in the liver. Subsequently, adult helminths migrate through the circulation to end with launching in the intestinal or the vesical venous plexus depending on the Schistosome species. However, the disease is triggered by the granulomatous body's reactions against the egg, not the adult helminth (Hams et al., 2013; El-Aal et al., 2015).

In S. mansoni infections granulomas are pathological lesions formed of masses of immune cells that target mainly the damage of the ova stage. Later, a process of tissue fibrosis initiates due to the stimulation of hepatic stellate cells. This hampers blood circulation in the affected parts and can 
be fatal due to the rupture of distended vessels in intestinal schistosomiasis (Raia et al., 1991). Fibrosis is assumed to be a reversible process while cirrhosis is irreversible. In this context, El-Hawary et al. (2018) assumed the importance of preventing the progression of fibrosis to cirrhosis.

Praziquantel (PZQ) has been described as the definite anti-bilharzial treatment for a long time ago to eliminate the parasite burdens; however, several studies stated its feeble outcome on the immature stages (El-Lakkany et al., 2012). In addition, PZQ showed an inadequate influence on liver fibrosis (Almeer et al., 2018). Curcumin, the bright yellow product, is an extract from the plant's species Curcuma longa which is a member of the ginger family. Besides being a spice and a cosmetic it has been associated with antioxidant and anti-inflammatory effects. Curcumin is thought to guard against lipid peroxidation and the oxidative damage of the DNA by the free radicals. This anti-inflammatory impact is most likely mediated through its capability to hinder cyclooxygenase-2, lipoxygenase, and the inducible nitric oxide synthase enzymes (Menon \& Sudheer, 2007; Rao, 2007).

BAX molecules are defined as pro-apoptotic within the BCL-2 family members. BAX expression increases in the cells after receiving an apoptotic stimulus and acts through the release of cytochrome-c (Korsmeyer et al., 2000); however, p53 directly regulates the production of BAX molecules attributable to DNA damage (Basu \& Haldar, 1998). The vital clinicopathological role of BAX in complicated schistosomiasis with colorectal cancer has been described by Yang et al. (2021). Additionally, recognition of apoptosis has been recently regarded as a vital target in the anti-schistosomes treatments (Wei et al., 2005; Lee et al., 2014).

IL17 is a pro-inflammatory mediator that promotes fibrosis and was found to promote the pathology of Schistosoma mansoni infection in the livers of murine models (Sun et al., 2020). Interestingly, S. mansoni infection was reported to alleviate collagen-induced arthritis via suppression of IL-17A. Also, Zhang et al. (2015) demonstrated its anti-fibrotic effect in schistosomiasis infection, a finding that may elect IL-17A as a valuable marker in the current therapeutic trial. The generation of host Th17 cells is suggested to be primarily induced by the schistosome egg antigens after the parasitic infection. The dynamics of Th17 cells during schistosomiasis infection results from the cohesive impact of stimulatory and inhibitory factors endorsed by the parasite (Wen et al., 2011). However, El-Aal et al. (2015) determined the poor control of chronic infection by unbalanced Th1/Th2 where Th2 almost dominates, with progressing lower Th 17 cells (Wen et al., 2011).

EGF was reported to increase the synthesis of DNA and proteins in addition to protein phosphorylation in S. mansoni worms (You et al., 2011). Therefore, EGF is considered to provide the parasite with the nutrients necessary for its growth and development (Vicogne et al., 2004).

In this study, we aimed to inspect the impact of curcumin with or without praziquantel on the pathological evidence and immature stages of the Schistosomes in the livers of the murine models using ISHAK scores and the immune-reactive pattern (IRS) scores of the immunohistochemical patterns of the three biomarkers BAX, IL17A, and EGF in tissue cut sections.

\section{MATERIALS AND METHODS}

\section{Animals}

Swiss albino male mice, 7 weeks old laboratory-bred in Theodor Bilharz Research Institute (TBRI). The housing of the animals was performed regarding the animal welfare regulations involving temperature $(25 \circ \mathrm{C})$, humidity, adequate ventilation, photoperiod of $12 \mathrm{~h}$ light $/ 12 \mathrm{~h}$ darkness, wellstructured formula, and daily cleaning of their cages from the fecal pellets and any dead bodies if present by a welltrained technician. Animals were exposed to regular veterinary examinations for common murine infections.

All procedures were implemented in regard to the guidelines of Clinical and Laboratory Standards Institute (CLSI) and were approved by the committee for animal ethics at TBRI and the ethical committee of the Cairo UniversityInstitutional Animal Care and Use Committee (CU- IACUC) and was registered by $\mathrm{CU} / \mathrm{III} / \mathrm{F} / 6 / 20$.

\section{Parasite cycle}

The laboratory preservation of $S$. mansoni (the Egyptian Strain) life cycle was achieved using Biomphalaria glabrata snails and Swiss albino mice. Each mouse was infected once with 200 cercariae.

\section{Experimental design}

The sample size was planned using the statistical analysis of the ANOVA test. Animals in the experiment were divided into four groups each of 12 mice. The following groups were incorporated in the study: group (1), animals were infected and treated with only curcumin; group (2), infected and received combined treatment (PZN + curcumin); group (3) infected and treated with praziquantel (PZQ), and group (4), infected and administrated distilled water and normal diet (positive control). Four animals from each group were sacrificed for specimen collection and processing on days 14 , 21 , and 35 post-treatments (P.T.).

\section{Infection}

Cercariae were shed from the infected snails and were quantified for infection of the experimental animals. During infection, all animals were locally anesthetized and their abdomens were shaved. A ring (one $\mathrm{cm}$ diameter) was located on a wet shaved area where suspensions of 200 cercariae were placed in the metal ring and permitted 30 $\min$ to penetrate.

\section{Treatment}

Praziquantel (PZQ) (Biltricide ${ }^{\circledR}$, Bayer, Germany) tablets were crushed into powder and then dissolved in distilled water. PZQ was received at a dose of $450 \mathrm{mg} / \mathrm{kg}$ body weight per day (Muchirah et al., 2012) and curcumin (Sigma-Aldrich, Co. St. Loius, MO, USA) was given as a daily oral dose of $600 \mathrm{mg} /$ $\mathrm{kg}$ body weight. Both lines of treatments were given at the fourth week post-infection continuing to the ninth week (the dose were given to three groups until 14, 21 and 35 days P.T.).

\section{Histopathological examination}

The excised liver tissue was immersed in formalin $10 \%$ then processed in alcohol, xylene and embedded in paraffin blocks. Four sections were cut from each paraffin block by microtome at 5 microns thickness; one stained with hematoxylin and Eosin for routine histopathological examination. The other three were cut on positively charged slides for immunostaining using IL17 (\#ab79056), BAX (\#EPR18283), and EGF (\#ab259398) (Abcam, Cambridge, UK) (Carleton, 1980).

Inflammation in the liver biopsies was graded according to the ISHAK scoring system, these assign numbers to the severity of the necro-inflammatory features (interface hepatitis, confluent necrosis, parenchymal injury, and portal inflammation) and add the numbers to arrive at a grade that 
can range from 0 to 18 . The number of granulomas was counted per $\mathrm{cm}^{2}$ (Brunt, 2000).

\section{Immunohistochemical analysis}

Tissue cut sections were deparaffinized and exposed to antigen retrieval through hydrogen peroxide and protein blocking. Each slide was incubated with 1/150 diluted mouse monoclonal antibody in primary antibody diluents against IL17(\#ab79056), BAX (\#EPR18283) and EGF (\#ab259398) (Abcam, Cambridge, UK). Sections were washed in PBS and incubated with an avidin-biotin-peroxidase system for 30 minutes. The brown color was developed using diaminobenzidine (DAB) which was applied for 4-5 minutes then slides were washed in the buffer. Antigen binding sites were visualized then counterstaining with hematoxylin was done. Sections were washed in tap water then dehydrated in ascending grades of alcohol then a drop of Canada balsam was added and sections were covered by a glass cover. The digital images of the selected tissue preparations were photographed using Olympus DP26 digital net camera attached to an Olympus CX31 Microscope (Olympus Corp., Shinjuku City, Tokyo, Japan).

The immunoreactive score (IRS) gives a range of 0-12 as a product of multiplication between the mean positive cells proportion score (0-4) and mean staining intensity score $(0-3)$. Positive cells were quantified as a percentage of the total number of cells, and assigned to one of five categories: 0 , no positive cells; $1,<10 ; 2,10-50 ; 3,51-80 ; 4,>80 \%$. The staining intensity was sub-classified as 0 (No stain), 1 (weak), 2 (moderate), and 3 (strong).

\section{Statistical methods}

The collected data were carefully reviewed, coded, and introduced using the "Microsoft Office Excel Software" program (2010) for windows. The pre-coded records were then conveyed to the Statistical Package of Social Science Software program, version 23 to be statistically evaluated. Data were obtained in the form of range, mean, standard deviation (S.D.) for quantitative variables. Descriptive statistics were introduced in the form of the mean and the standard deviation (+/- S.D.) for the parametric numerical records. For the analytical statistics, the chi-square test between infected untreated animals and treated groups at days 14, 21, 35 P.T. was performed to evaluate the statistical significance, the $P$-value was regarded as significant at $<0.05$. The results were symbolized in tables and graphs.

\section{RESULT}

A pathological assessment of liver tissues of all groups was performed and revealed the presence of chronic granulomas discernable by the presence of inflammatory infiltrates formed mainly of lymphocytes and eosinophils. No steatosis, cholestasis, dysplasia, malignancy, or distraction in the lobular architecture was observed in both treated and untreated animals, (Figure 1).

There was a significant reduction in the total number of granulomas per $\mathrm{cm} 2$ at day 35 P.T. between the combination therapy $(P Z Q+$ curcumin) and controls $(P<0.05)$ whereas curcumin individually revealed mild reduction $(P>0.05)$. PZQ and the combination therapy (PZQ + curcumin) showed a significant reduction in the counts of the fresh granulomas with visible ova and the best outcomes were recorded at day 35 P.T. $(P<0.05)$ (Table 1$)$. However, curcumin had the highest counts in the fresh granulomas compared with all other treated animals.

\section{ISHAK score}

There was a significant reduction in the score of the portal inflammation concomitant with the three therapeutic potentials in $P Z Q$, curcumin, and the combination therapy (PZQ and curcumin) in comparison with the control group. All treated animals were of reduced disease activity if compared with the control $(P<0.05)$. The effects of PZQ on the interface hepatitis yielded a greater score if compared to the curcumin and the combination therapy while being statistically insignificant if compared with the control (ISHAK score=3).

When lobular inflammation was analyzed, there was no statistical difference between the combination therapy $(\mathrm{PZQ}+$ curcumin) and the control $(P>0.05)$ with ISHAK score $=0$ whereas there were higher records at day 35 in the case of curcumin and those mice treated with PZQ (ISHAK score $=1$ ). In addition, the confluent necrosis at day 35 P.T. in curcumin experienced lower values (ISHAK score $=0$ ) with statistical significance $(P<0.05)$ with the corresponding control mice (ISHAK score $=2$ ), followed by group1, treated with PZQ, and the combination therapy (PZQ + curcumin) (ISHAK score $=1$ ). Overall, at day 35 P.T., there was a significant reduction in the fibrosis score in all treated mice (ISHAK scores $=2$ ) if compared with their corresponding controls (ISHAK scores $=3)(P<0.05)$ (Table 1).

\section{Immune-reactivity scores (IRS) of different biomarkers}

\section{BAX immune-reactivity}

In the case of curcumin, we observed cytoplasmic expression of BAX biomarker in, respectively, $(50 \pm 7.01 \%, 50 \pm 6.1 \%$, and $65 \pm 2.1 \%)$ and $(5 \pm 0.7 \%, 20 \pm 3.1 \%, 40 \pm 3.4 \%)$ of the inflammatory cells and hepatocytes at day 14, 21, and 35 P.T. In the combined therapy, BAX expression was significantly strong in the inflammatory cells in the periovular granulomas $60 \pm 3.2-2.9 \%$ and $65 \pm 3.5 \%$ at day 14,21 , and 35 P.T. In case of PZQ, the relative number of BAX-positive inflammatory cells or less remained at a constant level of immune reactivity of $30 \pm 6.09 \%, 40 \pm 5.3-5.9 \%$ at day 14 th, 21 st, and 35 th-day P.T. The number of immune reactive hepatocytes were very low, reporting zero or non-significant values among BAX-negative inflammatory cells at day 14, 21 and 35 P.T. (Figure 2). Control mice at day 14, 21, and 35 P.T. showed mean percentages of BAX expression $5 \pm 2.3-4.2 \%$ of the inflammatory cells. On day 14 P.T. BAX-positive hepatocytes were found in $10 \pm 3.3 \%$. Consequently, the relative number of immune reactive hepatocytes became increased up to $30 \pm 1.4 \%$ and $40 \pm 2.8 \%$ on days 21 and 35 respectively (Figure 3 ). The detailed data and interpretations were presented in Table 2.

\section{IL-17A immune-reactivity}

In the case of curcumin, the inflammatory cells showed high immune reactivity $(40 \pm 4.1)$ at day 14 P.T. At days 21 and 35 P.T. IL-17A-positive inflammatory cells declined significantly to $20 \pm 2.3 \%$ and $10 \pm 2.1 \%$ respectively. The hepatocytes showed an overall decrease from $2 \pm 0.2 \%$ at Day 14 P.T. to $5 \pm 0.3-0.4 \%$ at Day 21 and 35 P.T. In the case of combined therapy PZQ +curcumin, the percentage of the cytoplasmic labeled inflammatory cells gradually, but significantly, increased from $5 \pm 1.5 \%$ at day 14 P.T. to a peak value of $30 \pm 2.3 \%$ at day 21 P.T. and subsequently declined significantly to $10 \pm 1.2 \%$ at day 35 P.T. Mice treated with PZQ showed a moderate increase in IL-17A expression $(50 \pm 4.5,40 \pm 5.7$, and $50 \pm 5.3)$ at day 14,21 , 35 P.T. while being lacked in the hepatocytes. In the case of control, at days 14 and 21 P.T. few IL-17A-positive inflammatory cell clusters were present, and single immune reactive cells 


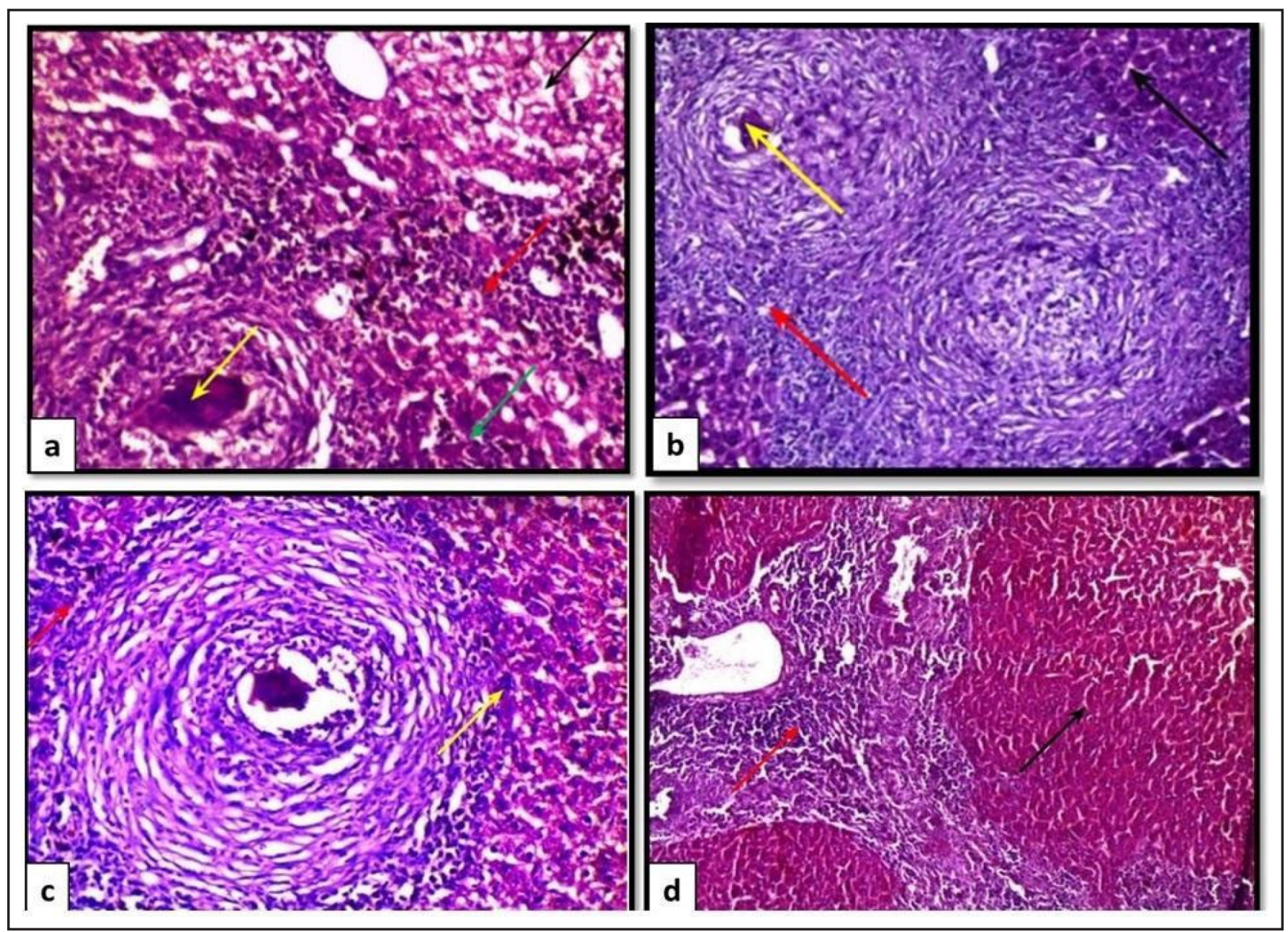

Figure 1. Hepatic granuloma in Swiss albino mice at day 35 P.T. in HE-stained liver sections. (a) Infected-PZQ treated mice: showing periovular granuloma (Yellow arrow) and hydropic degenerated hepatocytes (Black arrow). The portal tract showed increased portal lymphoplasmacytic cellular infiltrate (Red arrow) as well as interface hepatitis (Green arrow). (b) Infected-curcumin treated mice: the portal lymphoplasmacytic cellular infiltrates in the portal field; conversely if compared with the conventional treatment (PZQ), they were much less (red arrow). Note the ultimately calcified Bilharzial ova (Yellow arrow), the collagen deposition, and the preserved lobular and hepatocytes' architecture (Black arrow) (200x). (c) Infected-combined therapy treated mice: the portal lymphoplasmacytic cellular infiltrates (Red arrow) and the interface hepatitis was less frequent (Yellow arrow) in association with collagen deposition (400x). (d) Infected-untreated (control) mice: showing massive portal lymphoplasmacytic cellular infiltrate in the portal tract (Red arrow). However, neither the lobular architecture nor the hepatocytes showed any pathological changes (Black arrow) (200x).

were scattered inside the granulomas $(50 \pm 4.5$ and $40 \pm 5.7$ respectively). However, after day 35 P.T. the relative number of immune positive cells increased significantly $(65 \pm 5.8)$ (Figure 4). Hepatocytes stained by IL-17A were almost absent. Data and interpretations are shown in detail in Table 3.

\section{EGF immune-reactivity}

In the case of curcumin, the immune reactive inflammatory cells significantly decreased from $30 \pm 3.3 \%$ at day 14 P.T. to $10 \pm 1.6-2.1 \%$ at day 21 and 35 P.T., respectively. Membranous reactivity of the hepatocytes was almost absent at day 14; thereafter, EGF immune reactivity increased significantly until day 35 P.T. (10 $\pm 0.6-1.3 \%)$. In the case of combined treatment, the immune reactive cells formed clusters in $20 \pm 2.3-3.3 \%$ and $25 \pm 2.4 \%$ at day $14-, 21-$ and 35 -days P.T. respectively. EGFpositive hepatocytes were scattered in $3 \pm 0.2 \%$ and $5 \pm 1.2 \%$, among the EGF-negative hepatocytes at 14 and 21 P.T. respectively that however became moderately expressed in $10 \pm 1.3 \%$ of the hepatocytes at day 35 days. In the case of PZQ, the percentage of membranous labeled EGF inflammatory cells was increased to a peak value of $40 \pm 4.3 \%$ at day 14 P.T. then gradually, but significantly, reduced to $35 \pm 3.2$ at day 21 P.T. and $30 \pm 4.2 \%$ at day 35 P.T. The relative number of EGF immune positive hepatocytes showed a low value $(3 \pm 0.1$ $0.3 \%$ ) (Table 4 and Figure 5 ).

\section{DISCUSSION}

In the present study administration of curcumin limited the progression of fibrosis, lobular inflammation, and confluent necrosis. Similar to our results, curcumin has been suggested to limit the fibrogenic progression of experimental steatohepatitis by Vizzutti et al. (2010). El-Agamy, (2010) speculated that rats treated concurrently with curcumin showed a minimal degree of necrosis and inflammation. Also, Heritage et al. (2017), determined the lower values of alanine aminotransferase and the activity score of the nonalcoholic fatty liver disease in the presence of curcumin. In 


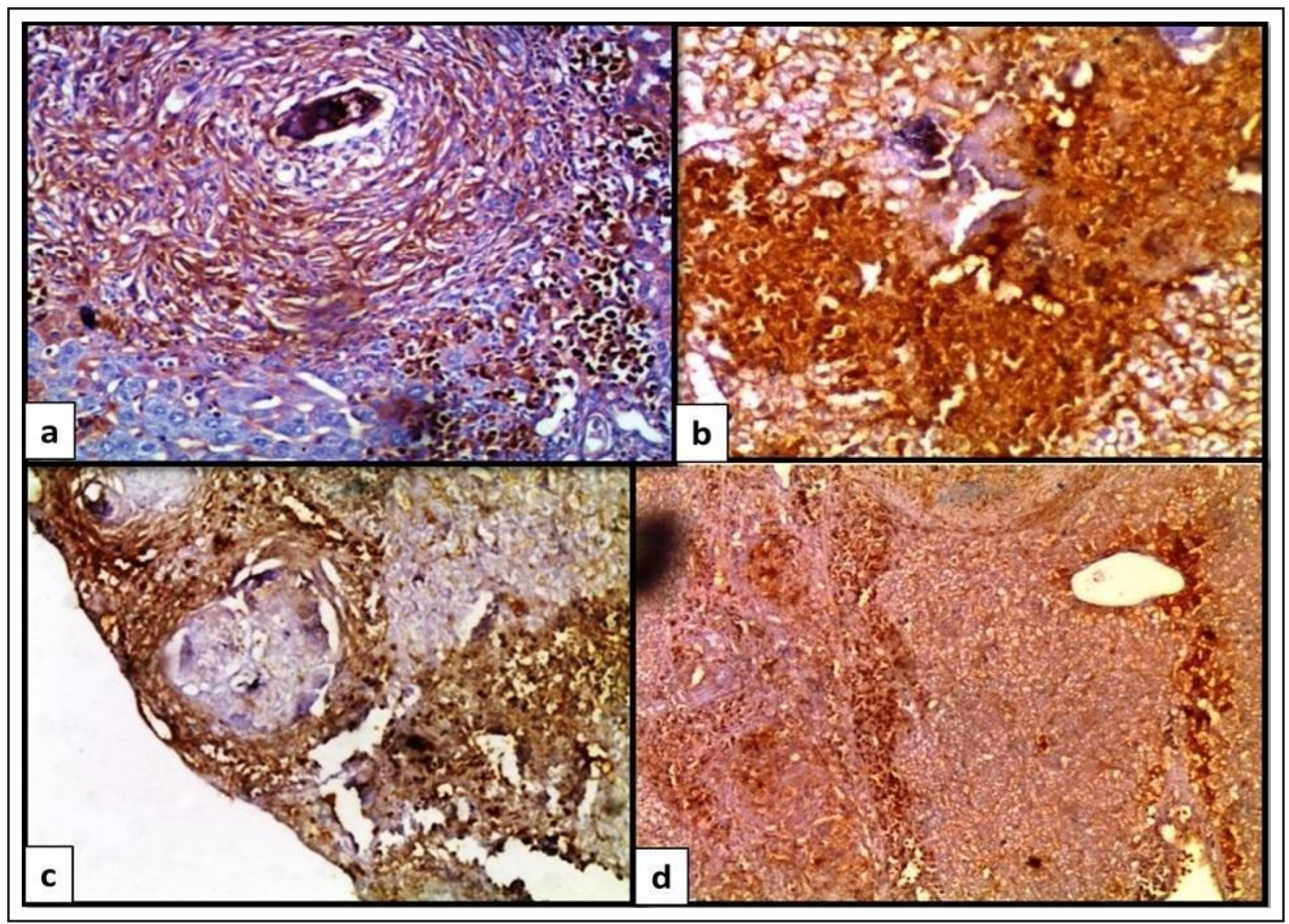

Figure 2. Detection of the cytoplasmic expression of BAX protein in the liver sections at day 35 P.T. (a): curcumin-treated group showing strong expression of BAX protein in $100 \%$ of the inflammatory cells and $80 \%$ of the hepatocytes (200x). (b): Combined treatment (PZQ+curcumin) treated group: showing clusters of inflammatory cells with strong BAX-positive stain while being relatively low in the hepatocytes (200x). (c): PZQ treated group showing strong positive stain in $50 \%$ of the inflammatory cells and low expressions in the hepatocytes (100x). (d): Mice infected and not treated (control) showing mild positive stain in $90 \%$ of the inflammatory cells and hepatocytes (200x).

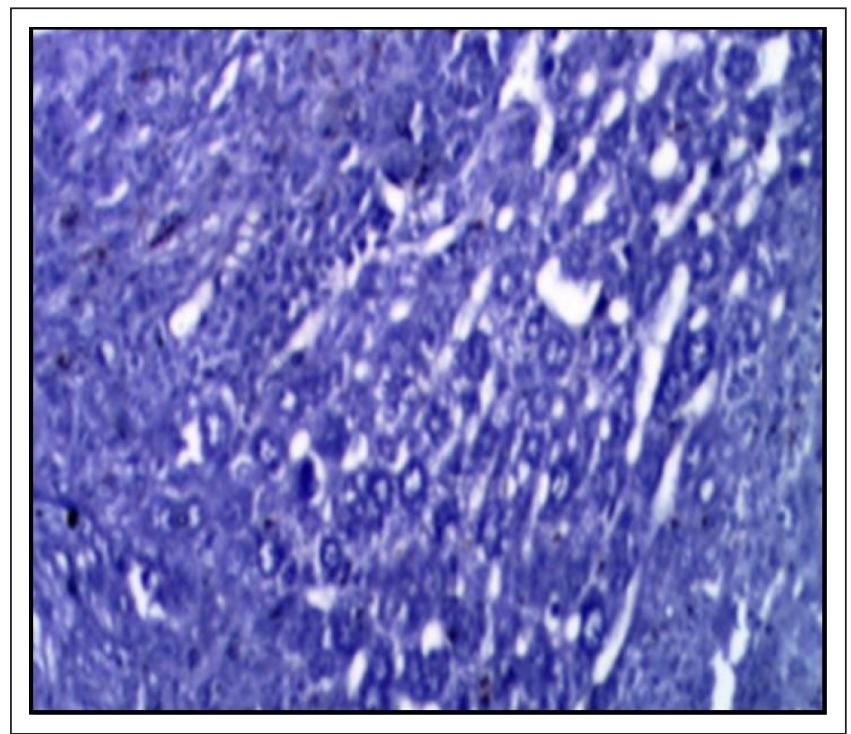

Figure 3. Control slide: the primary antibody staining step was omitted. this context, curcumin was found to lessen the hepatic inflammation via inhibition of NF-kB and the related pro-inflammatory genes (Leclercq et al., 2004). Interestingly, administration of curcumin was reported to be associated with higher hepatic storages of heme oxygenase-1, glutathione as well as superoxide dismutase (Li et al., 2016). Nevertheless, few case reports were documented for curcumin-induced liver injury (Lee et al., 2020).

In the current study, examination of liver sections of all infected and treated mice with various regimens revealed no remarkable differences in the activity and fibrosis ISHAK scores. Similar to our results Charoensuk et al. (2016) reported the effect of curcumin and PZQ in reducing periductal fibrosis and bile canalicular deformities.

Regarding our results, it had been shown that although the histopathological effect of curcumin is comparable to the control, the effect of conventional treatment PZQ and the combined therapy on the counts of periovular granulomas in the liver cut sections showed a remarkable reduction. From previous studies, PZQ treatment was found to reduce the burden of the adult worms and the mean counts of the daily produced miracidia (Lamberton et al., 2017). In 


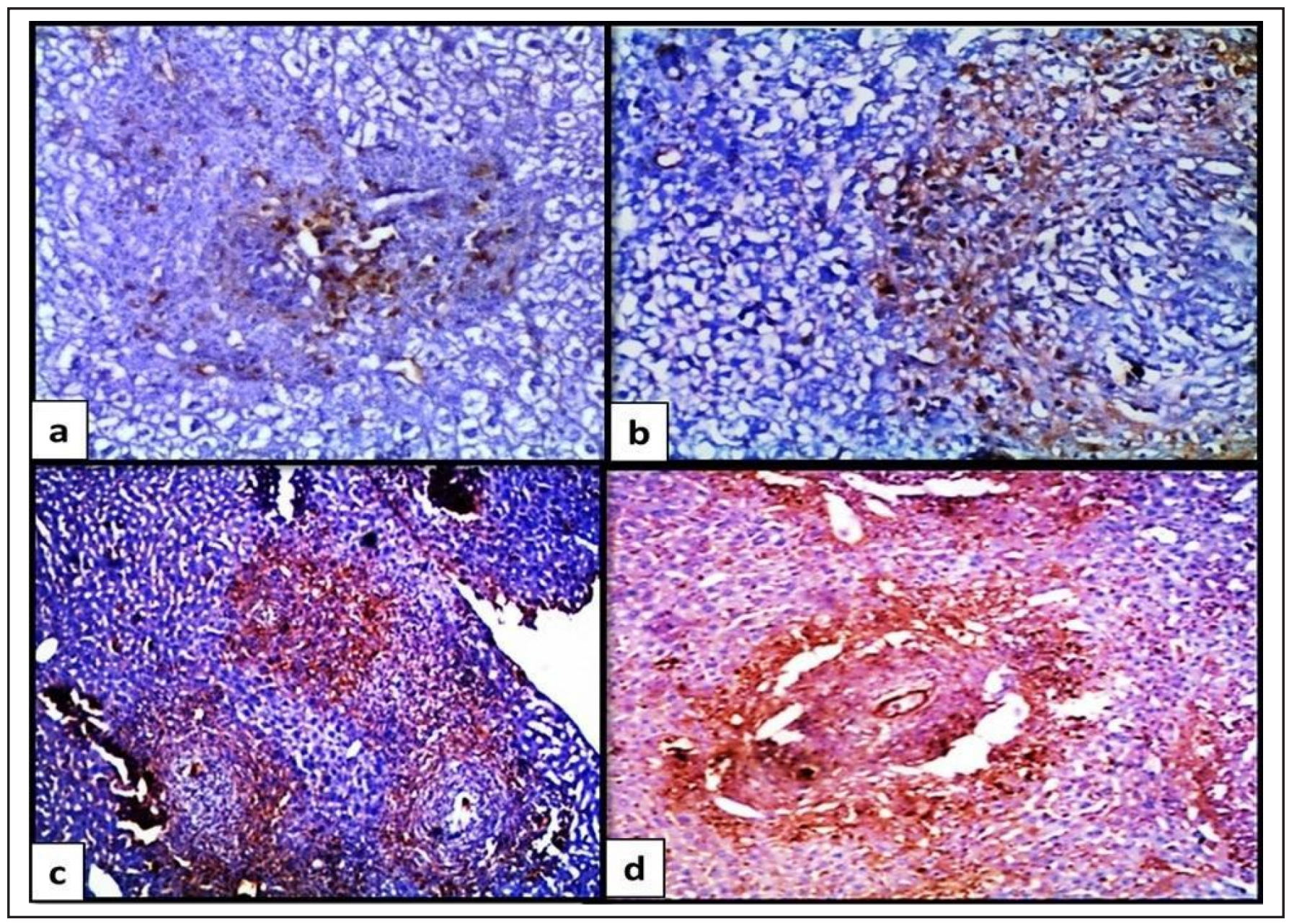

Figure 4. Detection of the cytoplasmic expression of IL-17A protein in the liver sections at day 35 P.T. (a): Curcumin-treated group: IL-17A positive staining was found to be only in the inflammatory cells and the hepatocytes were almost negative. (b): Combined therapy treated group showing IL-17A strongly positive inflammatory cells located in the periovular granuloma and mild expressions in the hepatocytes (200x). (c): PZQ treated control showing high expression in the inflammatory cells encompassing the Bilharzial ova and the hepatocytes that relatively lacked staining (100x). (d): Control group showing strongly positive stain in the inflammatory cells whereas the hepatocytes were of low expression (200x).

agreement with our results, Hussein et al. (2017) speculated the low potency of curcumin in reducing the burden of the parasite, granuloma size, and consequently the pathology of the affected liver. In contrast to our results, El-Ansary et al. (2007) speculated that curcumin extract is more potent in reducing egg count; however, the same authors determined the effectiveness of PZQ in lowering the worm burden.

In the current study, there was a significant increase in the expression of BAX using curcumin; similar to Araveti and Srivastava, (2019), that appeared to be a mitochondriadependent apoptotic pathway (Wang et al., 2009). Also, it has been reported that curcumin increases the $\mathrm{BAX} / \mathrm{BCl}-2$ ratio in breast cancers (Lv et al., 2014). In the current study, PZQ showed an increase in the expression of BAX similar to a previous study that reported oxidative stress resulting in apoptosis-like events leading to the death of the parasites (Giri \& Roy, 2016) due to the recruitment of the inflammatory cells adjacent to the bile ducts (Boonmars et al., 2008). Controls revealed lower expressions in BAX; in contrast to our study, Wei et al. (2005) reported significantly higher expression of BAX in their infected control group. Also, El Saftawy et al. (2021) reported increased pro-apoptotic signal in the cerebral tissues of murine models infected with Toxoplasma gondii.
In the present study, curcumin reduced the mean IRS of IL-17A and its mean percentage if compared to the infected untreated controls; similarly, Gouda et al. (2018) demonstrated the down regulatory impact of curcumin on IL $17 \mathrm{~A}$ mediated events. Interestingly, several studies suggested curcumin as a suppressor to IL-17A production through dampening the induced immune-mediated inflammation in H. pylori (Handono et al., 2015; Gouda \& Bhandary, 2018; Larussa et al., 2018). Lin et al. (2009) speculated that the anti-fibrotic events of curcumin are more prominent at lower concentrations and that it becomes pro-apoptotic at higher concentrations.

We, additionally, reported the increased expression of IL-17A in the periovular granulomas in the mice treated with PZQ; in agreement with Zou et al. (2011) who reported that PZQ facilitates the production of IL-17A through CD-8 cells. In this context, it is noteworthy to mention that decreasing IL-17 production using anti-IL-17 monoclonal antibody increased the sera levels of the anti-Bilharzia-specific antibodies providing partial guard against schistosomiasis infection (Wen et al., 2011).

In the present study, EGF was found to be suppressed promptly in the hepatocytes due to schistosomiasis infection. A similar observation was reported by (El Saftawy et al., 2020) 


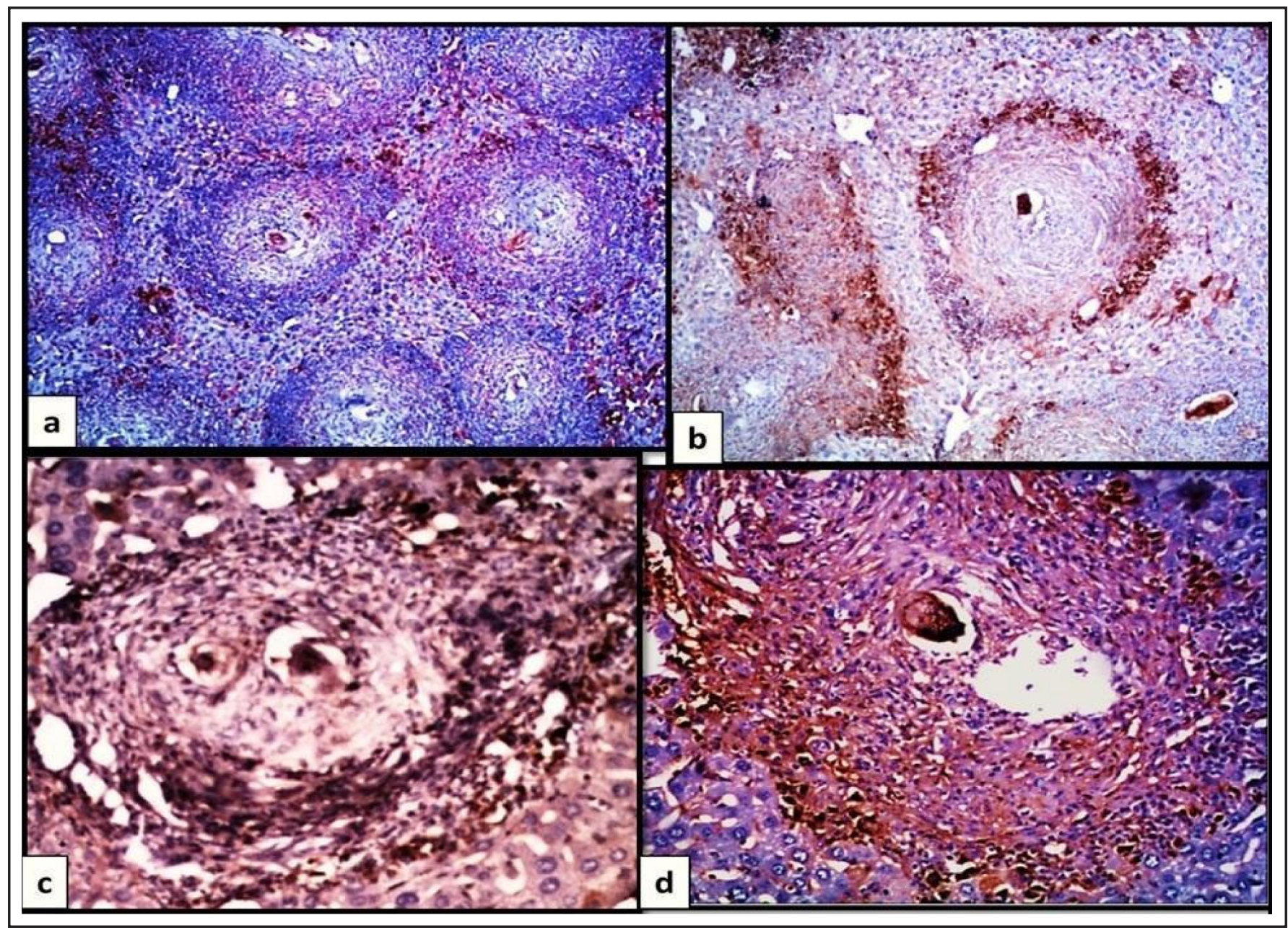

Figure 5. Detection of the membranous expression of EGF protein in the liver sections at day 35 P.T. (a) Curcumin-treated group showing moderate reactivity of the hepatocytes and the positive stain in periovular inflammatory cells (100x). (b) Combined therapy-treated group showing strong positive stain in the inflammatory cells and moderate immune- reactivity in the hepatocytes EGF antibody (200x). (c) PZQ-treated group: showing strong positive stain in the inflammatory cells surrounds the Bilharzial ova and mild reactivity in less than $5 \%$ of the hepatocytes $(100 \mathrm{x})$. (d) Positive control without treatment showing strong positively stained periovular granuloma (100x).

on the expression of insulin-like growth factors in murine cerebral tissues infected with Toxoplasma gondii. However, the combined treatment was associated with improvement in the expression of EGF in the hepatocytes at day 35 P.T.

Curcumin showed moderate expressions of EGF with regressed mean inflammatory cell percentages at day 14, 21, and 35 P.T. in the periovular granuloma; similar to our results, Ren et al. (2018) demonstrated the inhibitory effect of curcumin on cell proliferation by suppressing EGF on the molecular level. Also, it was observed to be a timedependent inhibitory effect similar to Ren et al. (2018) who indicated the same observation in the treatment of proliferative vitreoretinopathy.

\section{CONCLUSION}

The current study showed that PZQ +curcumin had the ultimate influence on granuloma reduction followed by PZQ as compared with the control. Curcumin showed notable improvement on ISHAK confluent necrosis score; also, PZQ +curcumin revealed a substantial reduction in the portal inflammation. Curcumin and PZQ + curcumin regimens promote $B A X$ expression in a time-dependent manner. IL$17 \mathrm{~A}$ and EGF showed lower expression in the periovular granulomas that comes in favor of an anti-inflammatory climate in the liver cut sections. This study significantly highlighted the potent synergistic contribution of curcumin to potentiate the therapeutic effects of the conventional treatment, $\mathrm{PZQ}$, in protecting the hosts from infection with lower immunopathology.

\section{Conflict of interests}

The author declares that they have no conflict of interests.

\section{REFERENCES}

Alemayehu, B., Tomass, Z., Wadilo, F., Leja, D., Liang, S. \& Erko, B. (2017). Epidemiology of intestinal helminthiasis among school children with emphasis on Schistosoma mansoni infection in Wolaita zone, Southern Ethiopia. BMC Public Health 17: 1-10. https://doi.org/10.1186/s12889-0174499-x

Almeer, R.S., El-Khadragy, M.F., Abdelhabib, S. \& Abdel Moneim, A.E. (2018). Ziziphus spina-christi leaf extract ameliorates schistosomiasis liver granuloma, fibrosis, and oxidative stress through downregulation of fibrinogenic signaling in mice. PloS ONE 13: e0204923. https://doi.org/ 10.1371/journal.pone.0204923 


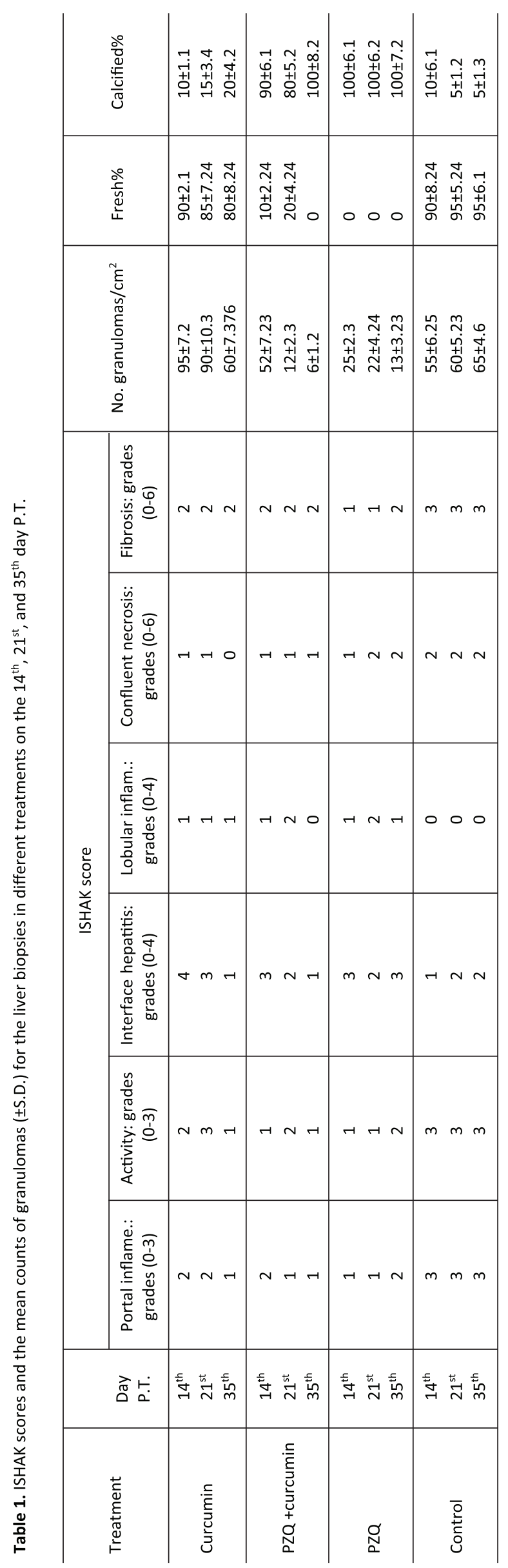

\begin{tabular}{|c|c|c|c|c|}
\hline 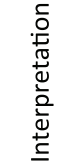 & 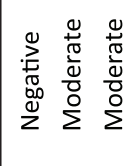 & 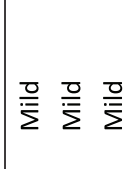 & 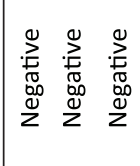 & $\stackrel{\frac{\partial}{\bar{\Sigma}}}{\bar{\Sigma}} \frac{\overline{0}}{\bar{\Sigma}} \frac{\overline{0}}{\bar{\Sigma}}$ \\
\hline$\sum_{\Sigma}^{\mathbb{\varpi}} \cong$ & $H \partial \theta$ & $\sim \sim \sim$ & $0 \begin{array}{ll}0 & -1\end{array}$ & $\sim \sim N$ \\
\hline $\begin{array}{l}0 \\
\stackrel{0}{0} \\
\stackrel{u}{\circ}\end{array}$ & $\rightarrow \sim N$ & $\begin{array}{lll}-1 & & \end{array}$ & $0 \begin{array}{ll}0 & -1\end{array}$ & $\sim \sim N$ \\
\hline 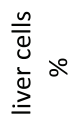 & 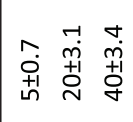 & 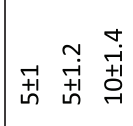 & 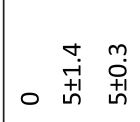 & 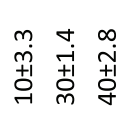 \\
\hline 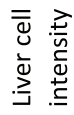 & 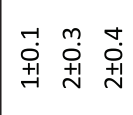 & 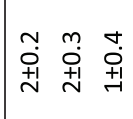 & 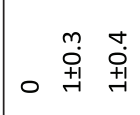 & 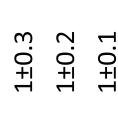 \\
\hline 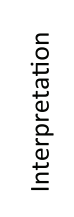 & 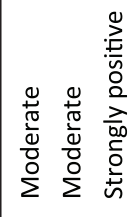 & 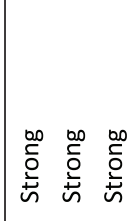 & 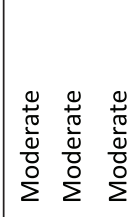 & 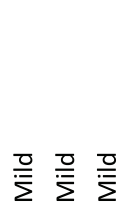 \\
\hline 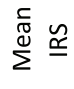 & 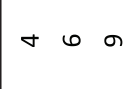 & $\begin{array}{lll}a & \sigma & \sigma\end{array}$ & $\sigma \sigma \sigma$ & 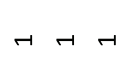 \\
\hline 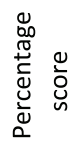 & $\sim \sim m$ & $m m m$ & $\sim \sim \sim$ & 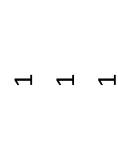 \\
\hline 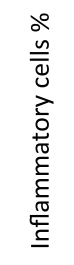 & 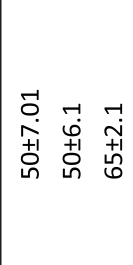 & 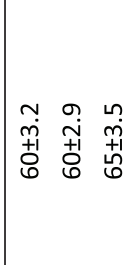 & 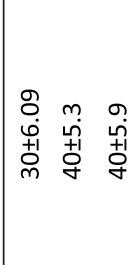 & 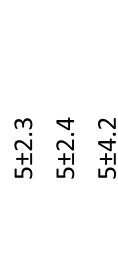 \\
\hline 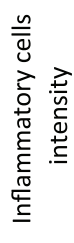 & 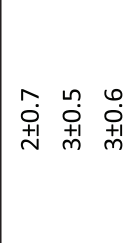 & 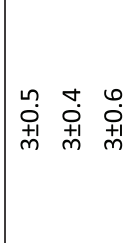 & 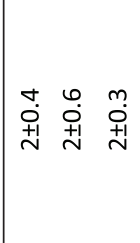 & 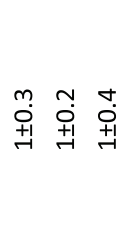 \\
\hline$\stackrel{-}{\circ}$ & 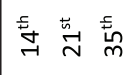 & 筧 & 尌营䓌 & 売 \\
\hline 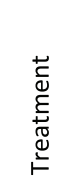 & 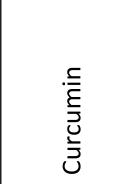 & 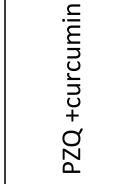 & 옴 & $\begin{array}{l}\overline{0} \\
\text { 홍 } \\
\text { o }\end{array}$ \\
\hline
\end{tabular}




\begin{tabular}{|c|c|c|c|c|}
\hline 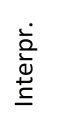 & 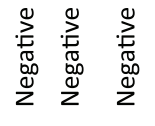 & 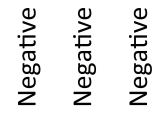 & 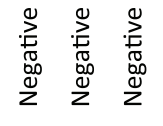 & 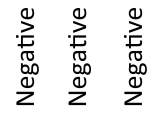 \\
\hline 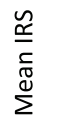 & 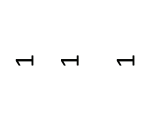 & 000 & $0 \quad 0 \quad 0$ & $\begin{array}{lll}-1 & 0 & -1\end{array}$ \\
\hline 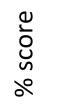 & $\begin{array}{rlr}-1 & -\end{array}$ & 000 & 000 & $\begin{array}{lll}-1 & 0 & -1\end{array}$ \\
\hline 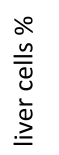 & 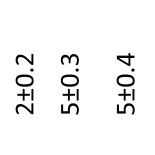 & 000 & 000 & 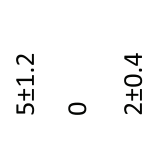 \\
\hline 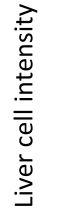 & 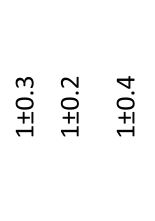 & 000 & 000 & 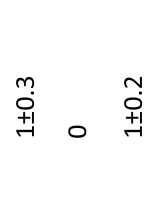 \\
\hline 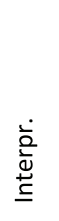 & 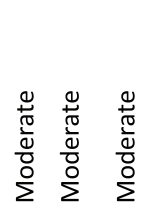 & 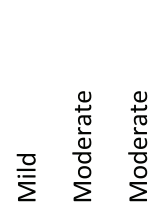 & 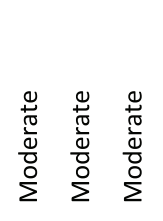 & 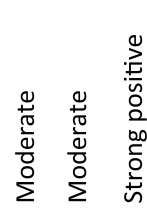 \\
\hline 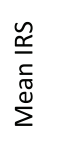 & 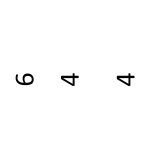 & $\sim 0 \sigma$ & 606 & $60 \mathrm{~m}$ \\
\hline 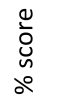 & $\sim \sim N$ & $\rightarrow \sim N$ & $N \sim N$ & $\sim \sim m$ \\
\hline 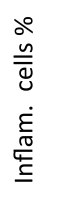 & 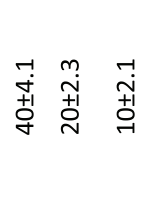 & 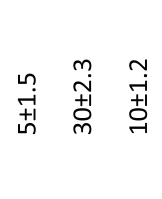 & 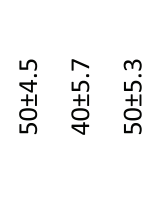 & 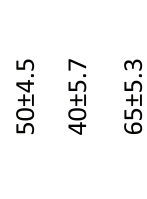 \\
\hline 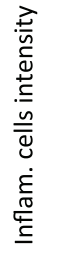 & 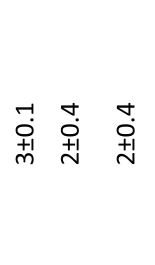 & 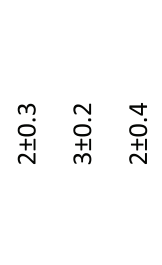 & 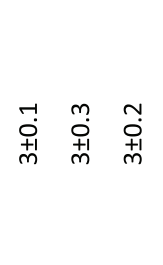 & 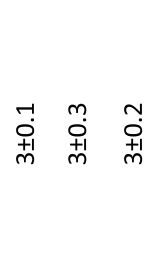 \\
\hline 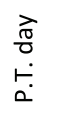 & 焉莣 & 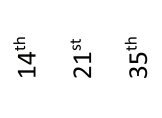 & 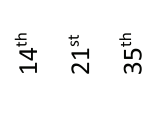 & 曾 \\
\hline 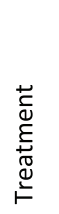 & 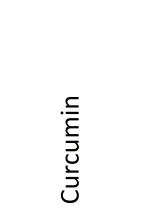 & 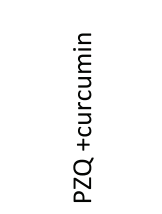 & 赵 & 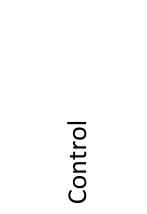 \\
\hline
\end{tabular}

\begin{tabular}{|c|c|c|c|c|}
\hline 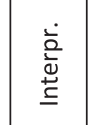 & 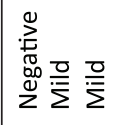 & 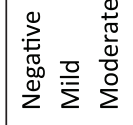 & 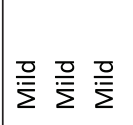 & 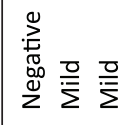 \\
\hline 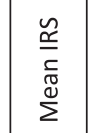 & $\forall \sim N$ & $\forall \sim \sigma$ & ન & $\forall \sim N$ \\
\hline 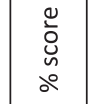 & $\rightarrow \sim N$ & $H \rightarrow N$ & $4 \rightarrow N$ & 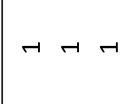 \\
\hline 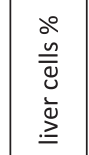 & 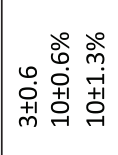 & 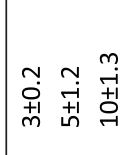 & 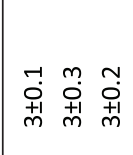 & 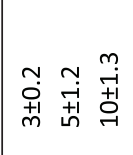 \\
\hline 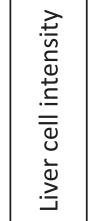 & $\begin{array}{lll}-1 & m & m \\
0 & 0 \\
+1 & 0 \\
+1 & +1 & +1\end{array}$ & 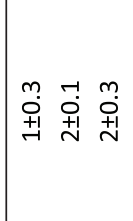 & 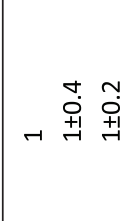 & 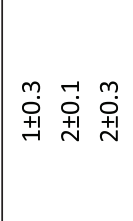 \\
\hline $\begin{array}{l}\stackrel{\dot{\bar{z}}}{\bar{v}} \\
\underline{\underline{E}}\end{array}$ & 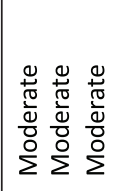 & 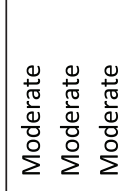 & 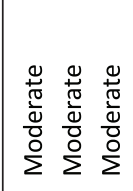 & 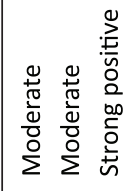 \\
\hline 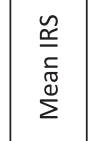 & $\sim \sim \sim$ & 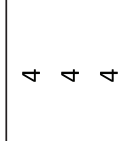 & 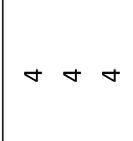 & $\theta+\sigma$ \\
\hline 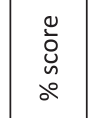 & $\sim \sim \sim$ & $\nabla \sim N$ & $\sim \sim \sim$ & $\nabla \sim m$ \\
\hline 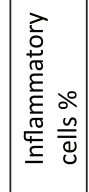 & 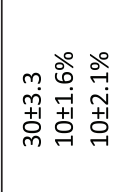 & 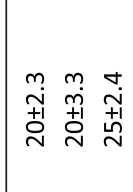 & 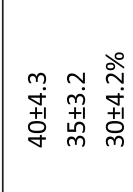 & 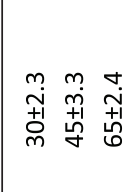 \\
\hline 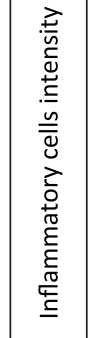 & 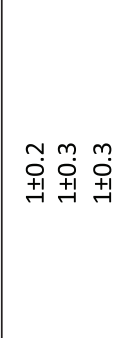 & 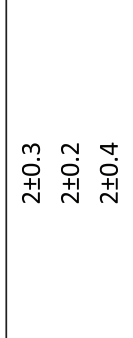 & 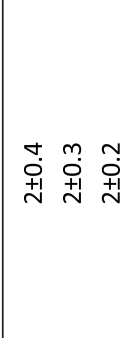 & 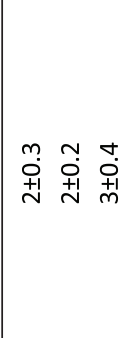 \\
\hline 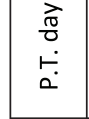 & 番 & 曾 & 曾 & 孚营点 \\
\hline 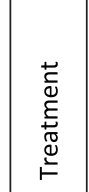 & 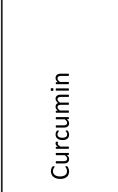 & 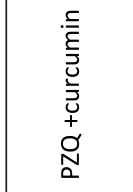 & 옴 & $\begin{array}{l}\text { 홈 } \\
\text { cे }\end{array}$ \\
\hline
\end{tabular}


Alzaylaee, H., Collins, R.A., Rinaldi, G., Shechonge, A., Ngatunga, B., Morgan, E.R. \& Genner, M.J. (2020). Schistosoma species detection by environmental DNA assays in African freshwaters. PLoS Neglected Tropical Diseases 14: e0008129. https://doi.org/10.1371/journal. pntd.0008129

Araveti, P.B. \& Srivastava, A. (2019). Curcumin induced oxidative stress causes autophagy and apoptosis in bovine leucocytes transformed by Theileria annulata. Cell Death Discovery 5: 1-14. https://doi.org/10.1038/s41420-019-01808

Basu, A. \& Haldar, S. (1998). The relationship between Bcl2, $\mathrm{BAX}$ and p53: consequences for cell cycle progression and cell death. Molecular Human Reproduction 4: 1099-1109. https://doi.org/10.1093/molehr/4.12.1099

Boonmars, T., Srirach, P., Kaewsamut, B., Srisawangwong, T., Pinlaor, S., Pinlaor, P., Yongvanit, P. \& Sithithaworn, P. (2008). Apoptosis-related gene expression in hamster opisthorchiasis post praziquantel treatment. Parasitology Research 102: 447-455. https://doi.org/10.1007/s00436-0070783-5

Brunt, E.M. (2000). Grading and staging the histopathological lesions of chronic hepatitis: the Knodell histology activity index and beyond. Hepatology 31: 241-246. https://doi.org/ 10.1002/hep.510310136

Charoensuk, L., Pinlaor, P., Wanichwecharungruang, S., Intuyod, K., Vaeteewoottacharn, K., Chaidee, A., Yongvanit, P., Pairojkul, C., Suwannateep, N. \& Pinlaor, S. (2016). Nanoencapsulated curcumin and praziquantel treatment reduces periductal fibrosis and attenuates bile canalicular abnormalities in Opisthorchis viverrini-infected hamsters. Nanomedicine: Nanotechnology, Biology and Medicine 12: 21-32. https://doi.org/10.1016/j.nano.2015. 10.005

Carleton, H.M. (1980). Carleton's histological technique. Drury, R.A.B. \& Wallington, E.A. (editors) $5^{\text {th }}$ edition. New York: Oxford University Press, pp. 140-142.

El Saftawy, E.A., Amin, N.M., Sabry, R.M., El-Anwar, N., Shash, R.Y., Elsebaie, E.H. \& Wassef, R.M. (2020). Can Toxoplasma gondii pave the road for dementia? Journal of Parasitology Research 2020: 1-13. https://doi.org/10.1155/2020/8859857

El Saftawy, E.A., Shash, R.Y., Aboulhoda, B.E., Arsanyos, S.F., Albadawi, E.A., Abou-Fandoud, S.M., Kamel, R.M. \& Amin, N.M. (2021). Insights into immunopathology and triggering of apoptosis in chronic cerebral toxoplasmosis using murine models. Tropical Biomedicine 38: 53-62. https:// doi.org/10.47665/tb.38.2.037

El-Aal, A.A.A., Emran, A.M., Al-Antably, A.S., El Saftawy, E.A., Bayoumy, I.R., Hassan, N.S. \& Badawi, M. (2015). Immunohistochemical pattern of $T$ lymphocytes population within bilharzial-associated bladder neoplasm microenvironment. International Journal of Immunopathology and Pharmacology 28: 209-217. https://doi.org/ 10.1177/0394632015584733

El-Agamy, D.S. (2010). Comparative effects of curcumin and resveratrol on aflatoxin $B_{1}$-induced liver injury in rats. Archives of Toxicology 84: 389-396. https://doi.org/10.1007/ s00204-010-0511-2

El-Ansary, A.K., Ahmed, S.A. \& Aly, S.A. (2007). Antischistosomal and liver protective effects of Curcuma longa extract in Schistosoma mansoni infected mice. Indian Journal of Experimental Biology 45: 791-801.
El-Hawary, S.S., Taha, K.F., Kirillos, F.N., Dahab, A.A., El-Mahis, A.A. \& El-Sayed, S.H. (2018). Complementary effect of Capparis spinosa L. and silymarin with/without praziquantel on mice experimentally infected with Schistosoma mansoni. Helminthologia 55: 21-32. https:// doi.org/10.1515/helm-2017-0055

El-Lakkany, N.M., Hammam, O.A., El-Maadawy, W.H., Badawy, A.A., Ain-Shoka, A.A. \& Ebeid, F.A. (2012). Antiinflammatory/anti-fibrotic effects of the hepatoprotective silymarin and the schistosomicide praziquantel against Schistosoma mansoni-induced liver fibrosis. Parasites \& Vectors 5: 1-14. https://doi.org/10.1186/1756-3305-5-9

Giri, B.R. \& Roy, B. (2016). Praziquantel induced oxidative stress and apoptosis-like cell death in Raillietina echinobothrida. Acta Tropica 159: 50-57. https://doi.org/10.1016/j. actatropica.2016.03.022

Gouda, M.M. \& Bhandary, Y.P. (2018). Curcumin down regulates IL 17A mediated p53 fibrinolytic system in bleomycin induced acute lung injury in vivo. Journal of Cellular Biochemistry 119: 7285-7299. https://doi.org/10.1002/ jcb. 27026

Gouda, M.M., Prabhu, A. \& Bhandary, Y.P. (2018b). IL-17A suppresses and curcumin up-regulates Akt expression upon bleomycin exposure. Molecular Biology Reports 45: 645-650. https://doi.org/10.1007/s11033-018-4199-3

Hams, E., Aviello, G. \& Fallon, P.G. (2013). The schistosoma granuloma: friend or foe? Frontiers in immunology 4: 89. https://doi.org/10.3389/fimmu.2013.00089

Handono, K., Pratama, M.Z., Endharti, A.T. \& Kalim, H. (2015). Treatment of low doses curcumin could modulate Th17/ Treg balance specifically on CD4+ T cell cultures of systemic lupus erythematosus patients. Central-European Journal of Immunology 40: 461-469. https://doi.org/10.5114/ ceji.2015.56970

Heritage, M., Jaskowski, L., Bridle, K., Campbell, C., Briskey, D., Britton, L., Fletcher, L., Vitetta, L., Subramaniam, V.N. \& Crawford, D. (2017). Combination curcumin and vitamin $\mathrm{E}$ treatment attenuates diet-induced steatosis in $\mathrm{Hfe}-/$ mice. World Journal of Gastrointestinal Pathophysiology 8: 6776. https://doi.org/10.4291/wjgp.v8.i2.67

Hussein, A., Rashed, S., El Hayawan, I., El-Sayed, R. \& Ali, H. (2017). Evaluation of the anti-schistosomal effects of turmeric (Curcuma longa) versus praziquantel in Schistosoma mansoni infected mice. Iranian Journal of Parasitology 12: 587 596.

Kabatereine, N.B., Brooker, S., Tukahebwa, E.M., Kazibwe, F. \& Onapa, A.W. (2004). Epidemiology and geography of Schistosoma mansoni in Uganda: implications for planning control. Tropical Medicine and International Health 9: 372-380. https://doi.org/10.1046/j.1365-3156.2003.01176.x

King, C.H. (2010). Parasites and poverty: the case of schistosomiasis. Acta Tropica 113: 95-104. https://doi.org/ 10.1016/j.actatropica.2009.11.012

Korsmeyer, S.J., Wei, M.C., Saito, M., Weiler, S., Oh, K.J. \& Schlesinger, P.H. (2000). Pro-apoptotic cascade activates $\mathrm{BID}$, which oligomerizes BAK or BAX into pores that result in the release of cytochrome c. Cell Death and Differentiation 7: 1166-1173. https://doi.org/10.1038/ sj.cdd. 4400783

Lamberton, P.H., Faust, C.L. \& Webster, J.P. (2017). Praziquantel decreases fecundity in Schistosoma mansoni adult worms that survive treatment: evidence from a laboratory lifehistory trade-offs selection study. Infectious Diseases of Poverty 6: 1-11. https://doi.org/10.1186/s40249-017-0324-0 
Larussa, T., Gervasi, S., Liparoti, R., Suraci, E., Marasco, R., Imeneo, M. \& Luzza, F. (2018). Downregulation of Interleukin-(IL-) 17 through enhanced Indoleamine 2, 3Dioxygenase (IDO) induction by curcumin: A potential mechanism of tolerance towards Helicobacter pylori. Journal of Immunology Research 2018: 3739593. https://doi.org/ $10.1155 / 2018 / 3739593$

Leclercq, I.A., Farrell, G.C., Sempoux, C., dela Peña, A. \& Horsmans, Y. (2004). Curcumin inhibits NF-êB activation and reduces the severity of experimental steatohepatitis in mice. Journal of Hepatology 41: 926-934. https://doi.org/ 10.1016/j.jhep.2004.08.010

Lee, B.S., Bhatia, T., Chaya, C.T., Wen, R., Taira, M.T. \& Lim, B.S. (2020). Autoimmune hepatitis associated with turmeric consumption. ACG Case Reports Journal 7: e00320. https:// doi.org/10.14309/crj.0000000000000320

Lee, E.F., Young, N.D., Lim, N.T., Gasser, R.B. \& Fairlie, W.D. (2014). Apoptosis in schistosomes: toward novel targets for the treatment of schistosomiasis. Trends in Parasitology 30: 75-84. https://doi.org/10.1016/j.pt.2013.12.005

Li, B., Wang, L., Lu, Q. \& Da, W. (2016). Liver injury attenuation by curcumin in a rat NASH model: an Nrf2 activationmediated effect? Irish Journal of Medical Science 185: 93100. https://doi.org/10.1007/s11845-014-1226-9

Lin, Y.L., Lin, C.Y., Chi, C.W. \& Huang, Y.T. (2009). Study on antifibrotic effects of curcumin in rat hepatic stellate cells. Phytotherapy Research 23: 927-932. https://doi.org/ 10.1002/ptr.2764

Lv, Z.D., Liu, X.P., Zhao, W.J., Dong, Q., Li, F.N., Wang, H.B. \& Kong, B. (2014). Curcumin induces apoptosis in breast cancer cells and inhibits tumor growth in vitro and in vivo. International Journal of Clinical and Experimental Pathology 7: 2818-2824.

Mawa, P.A., Kincaid-Smith, J., Tukahebwa, E.M., Webster, J.P. \& Wilson, S. (2021). Schistosomiasis morbidity hotspots: roles of the human host, the parasite and their interface in the development of severe morbidity. Frontiers in Immunology 12: 635869. https://doi.org/10.3389/fimmu. 2021.635869

Menon V.P. \& Sudheer A.R. (2007). Antioxidant and antiinflammatory properties of curcumin. In: The Molecular Targets And Therapeutic Uses of Curcumin In Health And Disease. Advances In Experimental Medicine And Biology. Aggarwal B.B., Surh YJ. \& Shishodia S. (editors). Boston: MA. Springer. pp. 105-125.

Muchirah, P.N., Yole, D., Kutima, H., Waihenya, R., Kuria, K.M. \& John, M. (2012). Determination of effective praziquantel dose in different mouse strains: BALB/c and Swiss mice in treatment of Schistosoma mansoni. Journal of Clinical Immnunology and Immunopathology Research 4: 12-21. https:/ /doi.org/10.5897/JCIIR12.004

Raia, S., Mies, S. \& Alfieri, F. (1991). Portal hypertension in mansonic schistosomiasis. World Journal of Surgery 15: 176187. https://doi.org/10.1007/BF01659051

Rao, C.V. (2007). Regulation of COX and LOX by curcumin. Advances in Experimental Medicine and Biology 595: 213-226. https://doi.org/10.1007/978-0-387-46401-5_9

Ren, Y.X., Ma, J.X., Zhao, F., An, J.B., Geng, Y.X. \& Liu, L.Y. (2018). Effects of curcumin on epidermal growth factor in proliferative vitreoretinopathy. Cellular Physiology and Biochemistry 47: 2136-2146. https://doi.org/10.1159/ 000491525

Sun, L., Gong, W., Shen, Y., Liang, L., Zhang, X., Li, T., Chen, T.T., $\mathrm{Hu}, \mathrm{Y}$. \& Cao, J. (2020). IL-17A-producing $\gamma \delta \mathrm{T}$ cells promote liver pathology in acute murine schistosomiasis. Parasites \& Vectors 13: 1-12. https://doi.org/10.1186/s13071-02004200-4
Tefera, A., Belay, T. \& Bajiro, M. (2020). Epidemiology of Schistosoma mansoni infection and associated risk factors among school children attending primary schools nearby rivers in Jimma town, an urban setting, Southwest Ethiopia. PloS ONE 15: e0228007. https://doi.org/10.1371/ journal.pone. 0228007

Vicogne, J., Cailliau, K., Tulasne, D., Browaeys, E., Yan, Y.T., Fafeur, V., Vilain, J.P., Legrand, D., Trolet, J. \& Dissous, C. (2004). Conservation of epidermal growth factor receptor function in the human parasitic helminth Schistosoma mansoni. Journal of Biological Chemistry 279: 37407-37414. https://doi.org/10.1074/jbc.M313738200

Vizzutti, F., Provenzano, A., Galastri, S., Milani, S., Delogu, W., Novo, E., Caligiuri, A., Zamara, E., Arena, U., Laffi, G. et al. (2010). Curcumin limits the fibrogenic evolution of experimental steatohepatitis. Laboratory Investigation 90: 104-115. https://doi.org/10.1038/labinvest.2009.112

Wang, J.B., Qi, L.L., Zheng, S.D. \& Wu, T.X. (2009). Curcumin induces apoptosis through the mitochondria-mediated apoptotic pathway in HT-29 cells. Journal of Zhejiang University Science B 10: 93-102. https://doi.org/10.1631/ jzus.B0820238

Wei, P., Luo, D.D., Xiong, L.J. \& Zeng, L.L. (2005). Expression of hepatic $\mathrm{BCl}-2$ and $\mathrm{BAX}$ proteins in schistosome-infected mice and the role of pentoxifylline. Zhongguo ji sheng chong xue yu ji sheng chong bing za zhi= Chinese Journal of Parasitology Parasitic Diseases 23: 441-443.

Wen, X., He, L., Chi, Y., Zhou, S., Hoellwarth, J., Zhang, C., Zhu, J., Wu, C., Dhesi, S., Wang, X. et al. (2011). Dynamics of Th17 cells and their role in Schistosoma japonicum infection in C57BL/6 mice. PLoS Neglected Tropical Diseases 5: e1399. https://doi.org/10.1371/journal.pntd.0001399

Yang, X.G., Yang, J.W., Zhao, P.J., Cheng, W., Shi, H.B., Zhang, B., Fu, Q.C. \& Li, Y. (2021). Expression and clinicopathological significance of $\mathrm{Bcl}-2$ and $\mathrm{BAX}$ genes in colorectal cancer patients complicated with schistosomiasis. Zhongguo xue xi Chong Bing Fang zhi za zhi= Chinese Journal of Schistosomiasis Control 33: 148-153.

You, H., Gobert, G.N., Jones, M.K., Zhang, W. \& McManus, D.P. (2011). Signaling pathways and the host parasite relationship: Putative targets for control interventions against schistosomiasis: Signaling pathways and future anti schistosome therapies. BioEssays 33: 203-214. https:// doi.org/10.1002/bies.201000077

Zhang, Y., Huang, D., Gao, W., Yan, J., Zhou, W., Hou, X., Liu, M., Ren, C., Wang, S. \& Shen, J. (2015). Lack of IL-17 signaling decreases liver fibrosis in murine Schistosomiasis japonica. International Immunology 27: 317-325. https:// doi.org/10.1093/intimm/dxv017

Zhao, Y., Yang, S., Li, B., Li, W., Wang, J., Chen, Z., Yang, J., Tan, H. \& Li, J. (2019). Alterations of the mice gut microbiome via Schistosoma japonicum ova-induced granuloma. Frontiers in Microbiology 10: 352. https://doi.org/10.3389/ fmicb.2019.00352

Zou, Q., Yao, X., Feng, J., Yin, Z., Flavell, R., Hu, Y., Zheng, G., Jin, J., Kang, Y., Wu, B. et al. (2011). Praziquantel facilitates IFN- $\gamma$-producing $\mathrm{CD}^{+} \mathrm{T}$ cells (Tc1) and IL-17-producing $\mathrm{CD} 8^{+}$ $T$ cells (Tc17) responses to DNA vaccination in mice. PLoS ONE 6: e25525. https://doi.org/10.1371/journal.pone. 0025525 